\title{
Immunomodulating property of MAPK inhibitors: from translational knowledge to clinical implementation
}

\author{
Mario Mandalà', Francesco De Logư ${ }^{2}$, Barbara Merelli ${ }^{1}$, Romina Nassini ${ }^{2}$ and Daniela Massi ${ }^{3}$
}

Treatment of metastatic melanoma was radically changed by the introduction of inhibitors of BRAF, an oncogene mutated in $40-50 \%$ of patients. Another area of advancement was the use of immunotherapy, and specifically, immune checkpoint inhibitors. There is compelling evidence that oncogenic BRAF, in addition to driving melanoma proliferation, differentiation and survival, induces T-cell suppression directly through the secretion of inhibitory cytokines or through membrane expression of co-inhibitory molecules such as the PD-1 ligands PD-L1 or PD-L2. Furthermore, the presence of oncogenic BRAF leads to an immune suppressive phenotype characterized by the presence of inhibitory immune cells such as regulatory T cells, myeloid-derived suppressor cells, or tumor-associated macrophages, which can in turn inhibit the function of tumor-infiltrating T cells. Growing evidence suggests that, in addition to their established molecular mechanism of action, the therapeutic efficacy of BRAF inhibitors and MEK inhibitors relies on additional factors that affect the tumor-host interactions, including the enhancement of melanoma antigen expression and the increase in immune response against tumor cells. Focus of the present review is to summarize the off target mechanisms of response to BRAF inhibitors and MEK inhibitors and the synergy between targeted therapy and immunotherapy as the biological source to open a window of strategic opportunities for the design of new exciting clinical trials.

Laboratory Investigation (2017) 97, 166-175; doi:10.1038/labinvest.2016.132; published online 19 December 2016

The therapy of metastatic melanoma $(\mathrm{MM})$ was radically changed by the introduction of inhibitors of BRAF, an oncogene mutated in $40-50 \%$ of patients. The BRAF inhibitors (BRAFi) proved to be more successful than conventional chemotherapy in the treatment of these patients in terms of activity and efficacy, achieving partial and complete remissions in many instances instances. ${ }^{1,2}$ Seminal clinical trials have shown that treatment of MM patients with BRAFi (vemurafenib, dabrafenib) is associated with improved response rate, progression free survival, and overall survival compared with conventional chemotherapy. ${ }^{1-3}$ However, while clinical responses to BRAFi may be dramatic, with some patients maintaining remission for several months or years, the median duration of response is $6-7$ months. ${ }^{1-3}$

This finding was the rationale for the introduction of MEK inhibitors (MEKi). ${ }^{4}$ The combination of BRAFi with MEKi was proposed as a strategy in MM patients to delay or even prevent the onset of resistance, without increasing the risk of developing secondary cancers. Three large, prospective, randomized clinical trials indicate that combined therapy is significantly more effective than either drug used alone and that resistance occurs at a significant later stage, proposing this combination as the new standard treatment in MM patients. ${ }^{5-7}$

Another area of improvement in melanoma treatment involves the use of immunotherapy, and specifically, the immune checkpoint inhibitors. Monoclonal antibodies targeting immunomodulatory molecules such as cytotoxic T-lymphocyte antigen-4 (CTLA-4) and programmed death receptor-1 (PD-1), represent a further advance, with objective response rates ranging between $15 \%$ and $40 \%$, respectively, when used as monotherapy, and up to $60 \%$ when combined in large randomized clinical trials. ${ }^{5,7,8}$ Although BRAF/MEK inhibitors are effective in xenograft models with impaired or depleted immune system, growing evidence suggests that the therapeutic efficacy of BRAFi and MEKi could rely on additional factors that affect the tumor-host interactions, including the enhancement of melanoma antigen expression and the increase in immune response against tumor cells., ${ }^{9,10}$ Consistently, preclinical data show that oncogenic BRAF

${ }^{1}$ Unit of Medical Oncology, Department of Oncology and Hematology, Papa Giovanni XXIII Cancer Center Hospital, Bergamo, Italy; ${ }^{2}$ Unit of Clinical Pharmacology and Oncology, Department of Health Sciences, University of Florence, Florence, Italy and ${ }^{3}$ Division of Pathological Anatomy, Department of Surgery and Translational Medicine, University of Florence, Florence, Italy

Correspondence: Associate Professor D Massi, MD, Division of Pathological Anatomy, Department of Surgery and Translational Medicine, University of Florence, Largo Brambilla, 3, Florence 50134, Italy.

E-mail: daniela.massi@unifi.it

Received 5 August 2016; revised 6 November 2016; accepted 7 November 2016 
contributes to immune evasion, and that targeting this mutation may increase the melanoma immunogenicity. ${ }^{11,12}$

Herein, we review the preclinical and clinical data supporting that BRAFi and MEKi are also immunomodulating drugs and suggest that combining targeted therapy with immunotherapy is potentially able to boost the immune responses or to overcome immune mediated mechanisms of resistance.

\section{Oncogenic BRAF as a Mechanism of Immunotolerance}

In the last years, extensive information emerged concerning the molecular basis of melanoma pathogenesis, progression, and response to therapy. There is evidence that cutaneous melanomas exhibit markedly elevated base mutation rates compared to nearly all other solid tumors, ${ }^{13,14}$ which is almost entirely attributable to increased abundance of the cytidine to thymidine $(\mathrm{C}>\mathrm{T})$ transitions a typical UV-lightinduced mutational signature. Among somatic mutations, activation of the mitogen-activated protein kinase (MAPK) pathway by oncogenic mutations was found in up to $90 \%$ of melanoma cases. ${ }^{15,16}$ Among them, BRAF mutations were demonstrated in $40-50 \%$ of melanomas. Most BRAF mutations localize to the kinase domain and increase the kinase activity of BRAF toward MEK. A valine-to-glutamate substitution in the glycine-rich loop is the most frequent BRAF mutation (V600E). This gain-of-function BRAF mutation accounts for more than $80 \%$ of the BRAF alterations described in melanoma, with alternative point mutations at the same position (V600D, V600K, V600R)

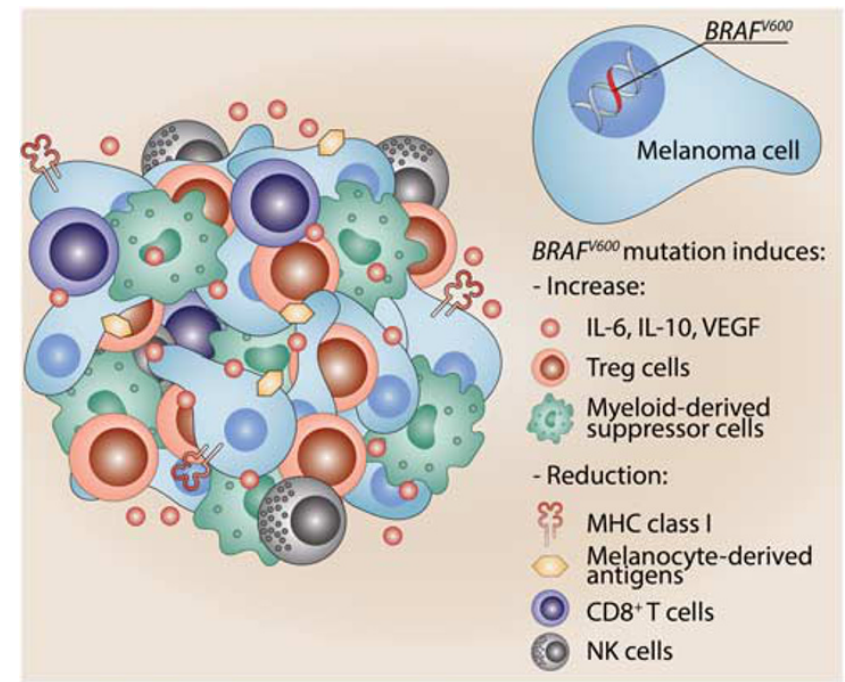

Figure 1 BRAFV600 mutation in melanoma cells increases the production of immunosuppressive factors (IL-10, VEGF, or IL-6) that, in turn, can promote recruitment of myeloid-derived suppressor cells and regulatory $T$ cells in the tumor microenvironment. Another immunosuppressive effect of mutant BRAF is related to the downregulation of MHC class I (MHC-I) molecules and decrease in CD8+ T/FoxP3+CD4+ T cell ratio and NK cells. contributing as following: $15 \%$ are $\mathrm{V} 600 \mathrm{~K}$, while V600R mutations constitute $\sim 3-5 \%$ of all BRAF mutations. ${ }^{17}$

There is evidence that oncogenic BRAF (BRAFV600) can lead to immunomodulation and in most cases immune escape in melanoma, ${ }^{11,12,18,19}$ and that blocking its activity via MAPK pathway inhibition leads to increased expression of melanocyte differentiation antigens. ${ }^{20,21}$

Four main mechanisms of immune escape mediated by oncogenic BRAF have been recognized: the first is mediated by melanoma cells through the release of immunosuppressive cytokines in the microenvironment; the second mechanism is mediated by cells of the microenvironment whose phenotype has been transformed from immunocompetent to immunosuppressive through the release of cytokines by tumor cells and by cells of the immune system; the third is mediated by the major histocompatibility complex (MHC) modulation in melanoma cells; the fourth is mediated by downregulation of tumor $\mathrm{Ccl} 2$ gene expression and decreased tumor CCL2 protein release. ${ }^{11,12,18,22,23}$

Twenty years ago, Sumimoto et al. ${ }^{11}$ demonstrated an essential role of the MAPK signaling on the production of various immunosuppressive factors (interleukin (IL)-10, VEGF, or IL-6) in melanoma cell lines with constitutively activated MAPK, due to BRAFV600 mutation. MEKi U0126 or RNA interference for BRAFV600 was able to reduce production of these immunosuppressive soluble factors from melanoma cells. These cytokines (IL-10, VEGF, or IL-6) can promote recruitment of inflammatory cell subsets such as myeloid-derived suppressor cells and regulatory $\mathrm{T}$ cells in the tumor microenvironment (Figure 1). In vitro experiments showed that melanoma cell lines harboring BRAFV600 mutation release the above reported cytokines in the supernatants, which in turn leads to an impaired production of inflammatory cytokines IL- 12 and tumor necrosis factor- $\alpha$ by dendritic cells upon lipopolysaccharide stimulation.

Furthermore, Khalili et al. ${ }^{12}$ extended the above observations demonstrating an indirect immune suppressive mechanism of oncogenic BRAF, essentially mediated by IL-1, which is released by melanoma cells. Ectopic expression of BRAFV600 in melanocytes can upregulate not only the expression of genes linked to oncogenic BRAF, including IL-6, IL-8, and VEGF, BRAFV600, but also significantly increases the transcription of IL- $1 \alpha$ and IL- $1 \beta$ genes as well as the release of IL-1 at the protein level (Figure 2). Intriguingly, BRAFi are able to block IL-1 protein production in human melanoma cell lines and in melanoma tumor biopsies derived from patients undergoing inhibitor treatment. IL-1, in turns, exerts its immune suppressive effect through melanomaassociated stromal fibroblasts, which exert potent inhibitory effects on melanoma antigen-specific CTLs following exposure to IL-1. Although the biological mechanism is far to be entirely elucidated, it seems that IL-1 leads to upregulate the transcription of several genes known to manifest immune suppression, such as COX-2, PD-L1 and PD-L2, which may contribute to the induction of a functional T-cell inhibition. 


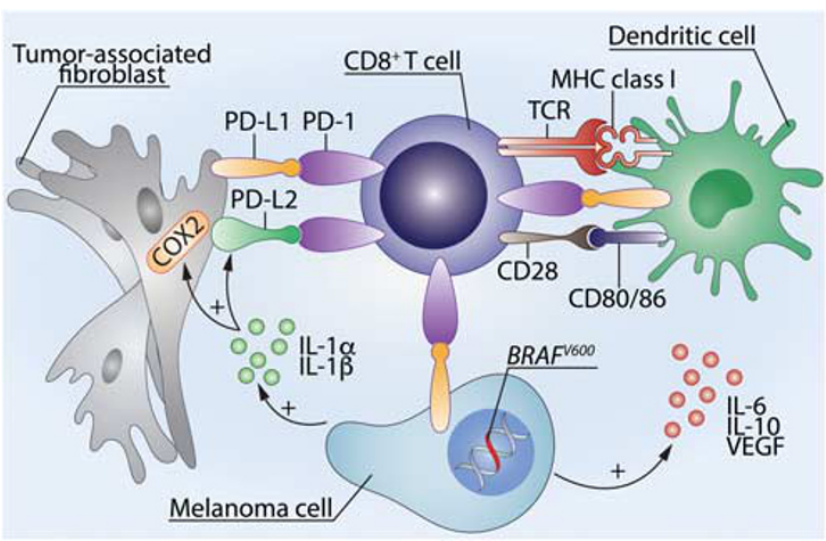

Figure 2 BRAFV600 mutation in melanoma cells increases the transcription of IL-1 $\alpha$ and IL-1 $\beta$ genes as well as the release of IL- 1 that exerts its immune suppressive effect through melanoma-associated stromal fibroblasts. These cells exert potent inhibitory effects on melanoma antigen-specific CTLs following exposure to IL-1. IL-1 upregulates the transcription of several genes known to manifest immune suppression, such as COX-2, PD-L1, and PD-L2, which may contribute to the induction of a functional T-cell inhibition (modified from Khalili et $a .^{12}$ ).

This study delineated an important and novel indirect link between oncogene activation in tumors and the resulting downstream effects on inflammation and immune suppression within the tumor microenvironment.

Another immunosuppressive effect of mutant BRAF is related to the downregulation of MHC class I (MHC-I) molecules by melanoma cells. MHC-I expression is reduced in melanoma cells overexpressing mutant BRAFV600. ${ }^{22,23}$ BRAFV600 mutation drives rapid and constitutive internalization of MHC-I from the cell surface and subsequent sequestration within endocytic compartments. Inhibition of BRAFV600 mutated human melanoma cells by BRAFi or MEKi decreased MHC-I internalization, resulting in less endolysosomal sequestration and increased surface expression, which in turn augments $\mathrm{CD} 8+\mathrm{T}$-cell recognition of tumor cells. Nevertheless there is no evidence, in cancer patients, that MHC is upregulated by BRAFi/MEKi.

Finally, by using transplantable, syngenic BRAF V600Edriven mouse melanoma in immunocompetent C57BL/6 mice, Knight et al. ${ }^{18}$ demonstrated that the BRAFi PLX4720 downregulates the tumor $\mathrm{Ccl} 2$ gene expression and decreases tumor CCL2 expression combined with a robust increase in CD8+ T/FoxP3+CD4+ T-cell ratio and NK cells. In addition, combination therapy with PLX4720 (BRAFi) and anti-CCL2 or agonistic anti-CD137 antibodies demonstrated significant antitumor activity suggesting that there is a synergistic effect combining BRAFi with immunotherapy.

In summary, the presence of oncogenic BRAF protein recapitulates several well-known immune suppressive mechanisms, including the inhibition of T-cell function, which are common to multiple cancers, with their presence frequently associated with poor patient prognosis. Oncogenic
BRAF induces T-cell suppression directly through the secretion of inhibitory cytokines such as IL-10, TGF- $\beta$, or VEGF, or through membrane expression of co-inhibitory molecules such as the PD-1 ligands PD-L1 or PD-L2. ${ }^{19}$ Furthermore, the presence of oncogenic BRAF leads to a immune suppressive phenotype characterized by the presence of inhibitory immune cells such as regulatory $\mathrm{T}$ cells, myeloid-derived suppressor cells, or tumor-associated macrophages, which can in turn inhibit the function of tumorinfiltrating $\mathrm{T}$ cells.

\section{BRAFI and MEKI Influence the Tumor-Host Interaction}

There is strong preclinical evidence that BRAFi and MEKi affect the tumor-host interactions, including the enhancement of melanoma differentiation antigens (MDAs) expression, eliciting the immune response against tumor cells. Although several preclinical data suggest that oncogenic BRAF downregulates MDAs, the underlying mechanism is far to be entirely elucidated.

Wellbrock et al. ${ }^{24}$ suggested that the biological mechanisms behind MDAs suppression by oncogenic BRAF expression relies on the interaction between MAPK and the microphthalmia-associated transcription factor (MITF), with the release of transcriptional repression when the MAPK pathway is blocked and subsequent expression of MITF targets, MDAs such as MART-1, gp100, TRP-1, and TRP-2. Nevertheless, there is an inherent complexity in the connection between BRAF and MITF. BRAF downregulates MITF through phosphorylation in ERK-dependent manner, but it also stimulates MITF transcription in BRN2-dependent manner. This tight dependence is important in melanocytes since MITF is a master regulator of melanocytes, intrinsically essential for melanocytes and melanoma cell survival and proliferation. Its mechanism of action depends on protein levels; differentiation functions of MITF occur at high protein concentrations, and accordingly, high levels of MITF are mainly anti-proliferative in melanoma cells. Nevertheless, low levels of MITF expression are necessary for proliferation and survival of melanoma cells because MITF regulates genes such as CDK2 and BCL-2, respectively.

In the context of oncogenic BRAFV600, BRAFi and MEKi lead to increased expression of MDAs, conferring enhanced antigen specific recognition by cytotoxic T cells. ${ }^{20}$ However, the MDAs induction by BRAFi and or MEKi is heterogeneous and variability in MDAs has been described across different melanoma cell lines. Namely, although MDAs are proportionally upregulated in different cell lines, the level of induction varies significantly between different melanoma cell lines. This not unique behavior suggests that additional signaling pathways could be important to melanoma pathophysiology and may account for the variability in MDAs induction. As a consequence, in clinical trials, it would be critical to assay further genetic alterations and the status of different pathway activation beyond MAPK and correlate these findings with expression of MDAs, as significant 
heterogeneity may exist even among BRAF-mutant melanomas. The translational relevance is quite clear: in patients whose MDAs are modulated by BRAFi, the addition of immunotherapy would be most effective. In cases in which MDAs expression is minimally altered, additional signaling inhibitors should be explored to achieve maximal antigen expression.

Nevertheless, three main issues remain still to be elucidated: (i) what is the translational role of upregulated MDA during treatment with BRAFi?; (ii) what is the impact of MAPKi on tumor microenvironment?: (iii) does MEK inhibition play a deleterious effect on $\mathrm{T}$ cells in in vivo models?

Although blocking MAPK by BRAFi or MEKi leads to increase of MDAs expression, there is evidence that tumorspecific neoantigens are important for immune recognition, and neoantigen-specific T-cell reactivity forms a major 'active ingredient' of successful cancer immunotherapies. In other words, the role of non-mutated MDA remains to be elucidated Hence, from a translational point of view, upregulation of MDA does not translate automatically in a better recognition by the immune system since the immune response to patient-specific neoantigens that arises as a consequence of tumor-specific mutations, and emerging data suggest that recognition of such neoantigens is a major factor in the activity of clinical immunotherapies.

There is evidence that in vitro BRAFi do not compromise lymphocytes' function, and may even augment T-cell activation via paradoxical signaling through the RAS-GTP pathways. In contrast, MEK inhibition has a similar effect on MDAs but deleterious effects on $\mathrm{T}$ cells. The notion that BRAFi increase MDAs expression and elicit T-cell activation has been subsequently proven in vivo, with the clear demonstration that treatment with BRAFi or combined BRAFi/MEKi results in a more favorable tumor microenvironment, particularly the increase of MDAs and $\mathrm{CD} 8^{+} \mathrm{T}$-cell infiltrate. $^{9,10}$

Two independent groups provided the first clinical evidence of a potential synergy between BRAFi and immunotherapy, demonstrating an increased in melanoma antigen expression in melanoma samples along with a decrease in VEGF and the immunosuppressive cytokines IL-6 and IL-8, associated with an enriched T-cell infiltrates within 14 days of the initiation of BRAFi therapy. ${ }^{9,10}$ Evidence of cytotoxic activity by $\mathrm{CD}^{+} \mathrm{T}$ cells and NK cells is supported by granzyme $\mathrm{B}$ and perforin staining in parallel to the increase inflammatory infiltrate. ${ }^{25}$

However, this more favorable microenvironment during BRAFi treatment seems to be counterbalanced by an increased expression of immunomodulatory molecules (namely, PD-1 and PD-L1) within 2 weeks of the initiation of BRAFi, a finding that suggests a potential immunemediated resistance mechanism to BRAF inhibition (Figure 3). Namely, although the infiltrating T cells in tumors of patients being treated with BRAFi demonstrate an activated

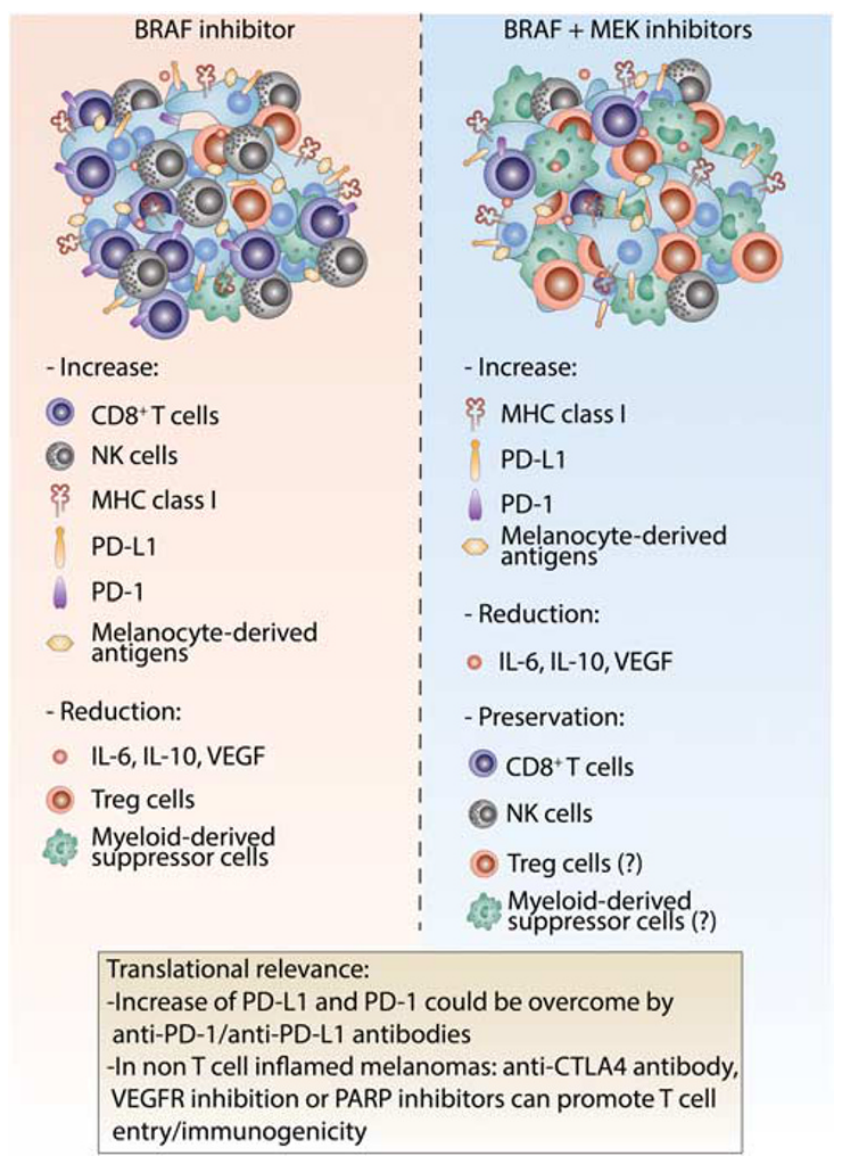

Figure 3 Upon BRAFi treatment, there is an increased expression of immunomodulatory molecules (namely, PD-1 and PD-L1) within 2 weeks of the initiation of therapy, a finding that suggests a potential immunemediated resistance mechanism to BRAF inhibition. Infiltrating $T$ cells in tumors of patients being treated with BRAFi also express high levels of PD-1 associated with overexpression of PD-L1 in melanoma cells, fibroblasts and immune cells in the microenvironment. The addition of MEKi does not seem to impair T-lymphocytes recruitment. However, T-cell antigen specificity and function have not been completely evaluated.

phenotype $\left(\mathrm{CD}^{+} /\right.$granzyme $\left.^{+}\right)$, they also express high levels of PD-1 associated with overexpression of PD-L1 in melanoma cells, fibroblasts, and immune cells in the microenvironment.

Regulation of PD-L1 expression by melanoma cells is indeed an area of intense investigation. PD-L1 can be induced by two mechanisms: the main is derived by microenvironmental signals, including interferon- $\gamma$, which is produced by activated $\mathrm{CD} 8^{+} \mathrm{T}$ lymphocytes; less frequently PD-L1 may be induced through oncogenic signaling. Ultimately, the interaction between PD-1/PD-L1 leads to immunosuppression, through inhibition of T lymphocytes' proliferation, induction of T-cell dysfunction, apoptosis of antigen-specific $\mathrm{T}$ cells as well as the promotion of the differentiation of $\mathrm{CD} 4^{+} \mathrm{T}$ cells into Foxp3+ regulatory T cells. These two mechanisms of PDL1 overexpression could be translational relevant in the context of BRAF-mutated melanomas. However, as combination of BRAFi and MEKi is now the standard treatment, 
Table 1 General properties of BRAF inhibitors (BRAFi) and immunotherapy

\begin{tabular}{lll}
\hline & Targeted therapy (BRAFi) & Immune modulating agents \\
\hline Target & Oncogenic BRAF & T-cell CD8 ${ }^{+}$ \\
Pharmacokinetics & Short (hours) & Long (weeks) \\
Pharmacodynamics & Short (hours-days) & Long (months-years) \\
What kills melanoma & Small molecules that turn off an oncogenic signal & The immune system that kills its targets anywhere in the body \\
Body distribution & Passive (blood distribution) & Active (T cells searching for antigen) \\
Memory & No & Yes \\
Timing of response & Days & Weeks
\end{tabular}

dissecting the in vivo influence of MEKi on immune cells is particularly important from a clinical and translational point of view. Although, in vitro, MEK inhibition has deleterious effects on $\mathrm{T}$ cells, recent data suggest that MEK inhibitors block growth of tumors in mice while promoting the effector phenotype of tumor-infiltrating $\mathrm{CD}^{+} \mathrm{T}$ cells. Furthermore, and most important, targeting these $\mathrm{T}$ cells by combining MEK inhibitor with anti-PD-L1 treatment resulted in a synergistic inhibition of tumor growth, including durable responses including complete regressions. ${ }^{26}$

Furthermore, recent data found no evidence for marked immunosuppressive effects in tumor-infiltrating lymphocytes (TILs) in the sequential biopsies taken from patients treated with combo BRAFi and MEKi, which appeared comparable to those in patients treated with BRAFi monotherapy. ${ }^{27,28}$ More importantly, in paired biopsies, the addition of MEKi to BRAFi did not result in significant reduction in immune infiltration of $\mathrm{CD}^{+}$and $\mathrm{CD} 8^{+}$lymphocytes in the early phase of treatment. Hu-Liescovan et al. ${ }^{29}$ have recently reported complementary results. By using a mouse model of syngeneic BRAFV600E-driven melanoma, the Authors tested the hypothesis that in vivo MEKi would enhance the antitumor activity of combined immunotherapy with the BRAFi. Complete tumor regression was observed with combination of BRAFi and MEKi with adoptive cell transfer, accompanied by increasing T-cell infiltration and cytotoxicity into tumors. The addition of MEKi to single-agent BRAFi increased tumor-associated macrophages and $\mathrm{T}$ regulatory cells as compared with BRAFi alone. Finally, the triple combination therapy resulted in increased melanosomal antigen and MHC expression and global immune-related gene upregulation.

Finally, we previously demonstrated that in BRAFi-treated MM patients, PD-L1 immunohistochemical expression in melanoma cells and absence of tumor-infiltrating mononuclear cells (TIMC) were significantly associated with shorter progression-free survival and melanoma-specific survival. $^{30}$ By multivariate analysis, absence of tumoral PD-L1 staining and the presence of TIMC were associated with a better response to treatment. ${ }^{30}$
Altogether, the above-reported data provide support for conducting trials, several of which are now ongoing, that combine MAPKi with immune checkpoint inhibitors in the hope of improving complete and durable response rates.

\section{Rational to Combine Immunotherapy and Targeted Therapy and Ongoing Clinical Trials}

In MM patients, two parallel strategies have been developed: combination of BRAFi and MEKi and immune checkpoint inhibitors. Although these two strategies have been developed in parallel and in a completely different way, targeted therapies and immunotherapy are two universes that collide, although difference remain, which, ultimately, increase the complementarity of these two approaches (Table 1). The optimal sequencing of these two different classes of systemic treatments is currently unknown. Among several open questions, there are at least three issues to be addressed: (i) toxicity of combination treatment; (ii) the optimal sequence; (iii) which drugs should be implemented.

Initial efforts using ipilimumab in combination with the BRAFi vemurafenib were limited by toxicity. In a phase 1 study, patients with MM with known BRAFV600E mutation received a 1-month run in with a BRAFi (vemurafenib) alone followed by four infusions of ipilimumab in combination with full doses of vemurafenib ( $960 \mathrm{mg}$ orally twice daily) in the first cohort, and lower dose of vemurafenib $(720 \mathrm{mg}$ twice daily) together with the full dose of ipilimumab in the second cohort. ${ }^{31}$ This trial was stopped early due to toxicity; specifically hepatotoxicity was observed in a substantial proportion of patients, consisting mainly of grade 2 or 3 elevations in liver function tests (LFT and bilirubin level).

Recently, Puzanov et al. reported a phase 1 study, which was undertaken to determine the safety of dabrafenib with and without trametinib and ipilimumab at the following doses: dabrafenib $100 \mathrm{mg}$ BID, trametinib $1 \mathrm{mg}$ QD and ipilimumab at the FDA-approved dose of $3 \mathrm{mg} / \mathrm{kg}$ every 3 weeks for 4 doses. Among 7 advanced melanoma patients 2 developed grade 3 colitis complicated by perforation. ${ }^{32}$ Therefore, accrual to the triple combination arm was stopped, while monotherapy with dabrafenib and ipilimumab 
Table 2 Ongoing clinical trials with targeted therapy combined to immunotherapy in advanced melanoma patients (from www. clinicaltrials.gov, updated on 8 August 2016)

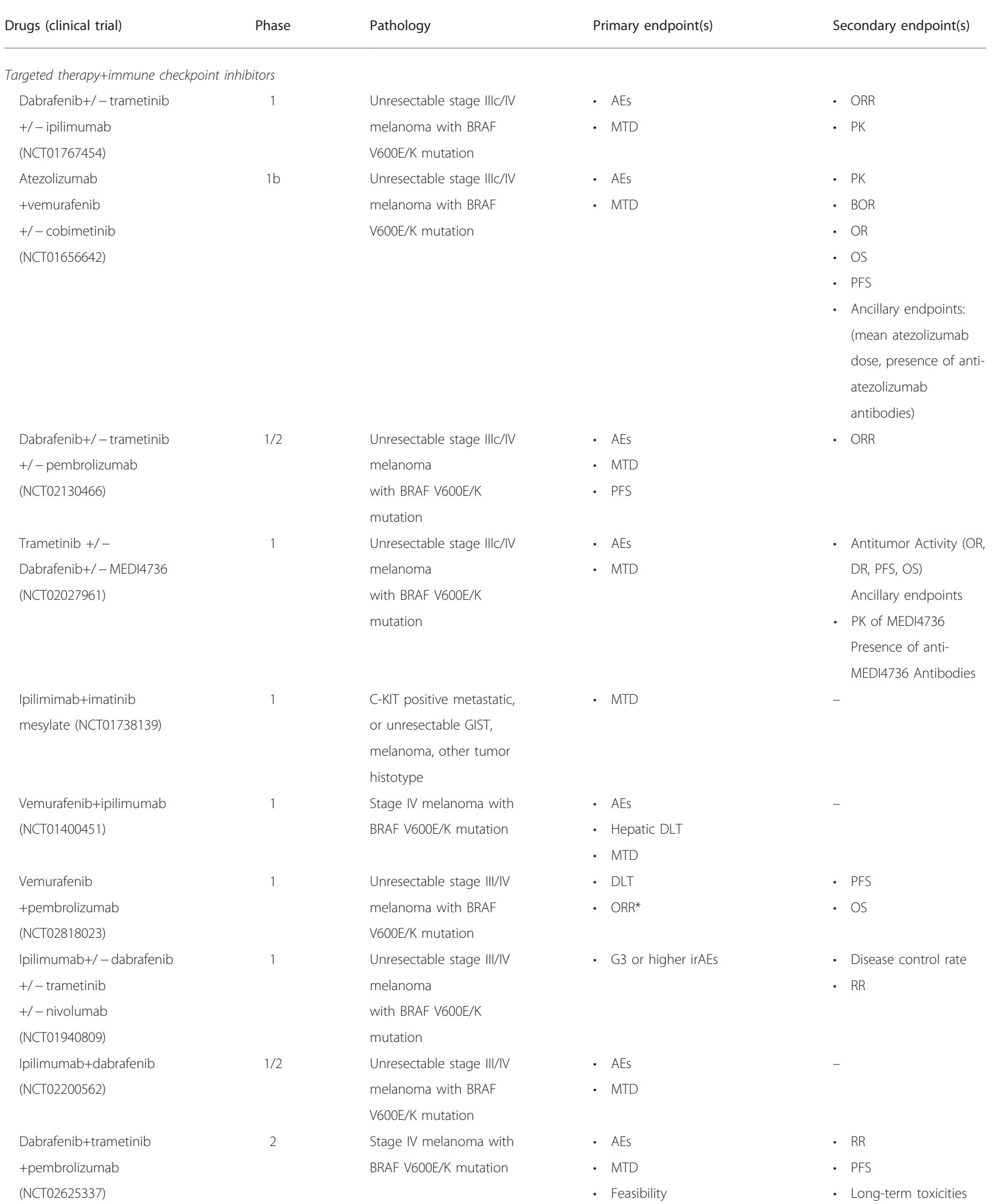


Table 2 Continued

\begin{tabular}{|c|c|c|c|c|}
\hline Drugs (clinical trial) & Phase & Pathology & Primary endpoint(s) & Secondary endpoint(s) \\
\hline Imatinib+pembrolizumab & $1 / 2$ & Unresectable stage III/ IV & - ORR & - Change in PD-1 and \\
\hline \multirow[t]{5}{*}{ (NCT02812693) } & & melanoma with c-KIT & & PDL-1 expression levels \\
\hline & & mutation/amplification & & - Incidence of AEs \\
\hline & & & & - OS \\
\hline & & & & - PFS \\
\hline & & & & - TTP \\
\hline \multicolumn{5}{|l|}{ Targeted therapy+cytokines } \\
\hline Vemurafenib+IL-2 & 2 & Stage IIIc/IV melanoma & - PFS & $\cdot$ ORR \\
\hline \multirow[t]{5}{*}{ (NCT01754376) } & & with BRAF V600E & & - OS \\
\hline & & mutation & & - Toxicity and safety of \\
\hline & & & & IL-2 and vemurafenib \\
\hline & & & & Ancillary endpoints \\
\hline & & & & - Biomarkers \\
\hline Vemurafenib+high dose & 4 & Stage IV melanoma with & - $\mathrm{CR}$ rate & - \\
\hline IL-2 (NCT01683188) & & BRAF V600E/K mutation & & \\
\hline Vemurafenib+IL-2 & $1 / 2$ & Unresectable stage III/IV & - AEs & - \\
\hline (infusional 96 h)+INF alfa- & & melanoma with BRAF & - MTD & \\
\hline 2b (NCT01603212) & & V600E/K mutation & - PFS & \\
\hline Vemurafenib+pegylated & $1 / 2$ & Unresectable stage III/IV & - AES & - ORR \\
\hline \multirow[t]{2}{*}{ IFN (NCT01959633) } & & melanoma with BRAF & & \\
\hline & & V600E/K mutation & & \\
\hline Vemurafenib+high-dose & $1 / 2$ & Unresectable stage III/IV & - AES & - PFS \\
\hline \multirow[t]{2}{*}{ INF alfa-2b (NCT01943422) } & & melanoma with BRAF & & - OS \\
\hline & & V600E/K mutation & & \\
\hline \multicolumn{5}{|l|}{ Targeted therapy $+T$ cell } \\
\hline Vemurafenib & 1 & Stage IV melanoma with & - AES & - \\
\hline +cyclophosphamide and & & BRAF V600E/K mutation & - MTD & \\
\hline \multicolumn{5}{|l|}{ fludarabine $+\mathrm{TIL}+$ high dose } \\
\hline \multicolumn{5}{|l|}{ IL-2 (NCT 01585415) } \\
\hline Vemurafenib+ACT with TIL & 2 & Unresectable stage III/IV & $\cdot$ ORR & - PFS \\
\hline infusion+high-dose IL-2 & & melanoma with BRAF & - Drop out rate & \\
\hline (NCT01659151) & & V600E/K/D mutation & & \\
\hline Vemurafenub+ACT+TIL & $1 / 2$ & Unresectable stage III/ IV & - AES & - Immune-related \\
\hline \multirow[t]{4}{*}{ infusion (NCT02354690) } & & melanoma with BRAF & - MTD & responses \\
\hline & & V600E/K mutation & & - ORR \\
\hline & & & & $\cdot$ OS \\
\hline & & & & - PFS \\
\hline
\end{tabular}

Abbreviations: $A C T$, adoptive cell transfer; $A E s$, adverse events; $B O R$, best overall response; $C R$, complete response; DLT, dose-limiting toxicity; $D R$, duration of response; IFN, interferon; IL-2, Interleukin-2; irAEs, immune-related adverse events; MTD, maximum tolerated dose; OR, overall response; ORR, objective response rate; OS, overall survival; PFS, progression-free survival; PK, pharmacokinetic; SAEs, serious adverse events; TIL, tumor infiltrating lymphocytes; TTP, time to progression.

combination is ongoing. A third trial is assessing the combination of the anti-PD-L1 antibody MEDI4736 at 3 or $10 \mathrm{mg} / \mathrm{kg}$ every 2 weeks, together with dabrafenib $150 \mathrm{mg}$ BID and trametinib $2 \mathrm{mg}$ QD. ${ }^{33}$ Patients have been enrolled according to the BRAF status into dose escalation cohorts (3+3 design), followed by dose expansion: BRAF mutant in 
Cohort A (MEDI4736+Dabrafenib+Trametinib); BRAF WT in Cohort B (MEDI4736+Trametinib) or Cohort C (sequential Trametinib $\rightarrow$ MEDI4736). Fifty patients were treated so far. Dose limiting toxicities (DLTs) were observed in 1 patient in Cohort A (reversible grade 3 thrombocytopenia) and one patient in Cohort B (reversible G3 choroidal effusion). No maximum tolerated dose (MTD) was identified; MEDI4736 $10 \mathrm{mg} / \mathrm{kg} \mathrm{q} 2 \mathrm{w}$ was selected for expansion in all cohorts. The most frequent drug-related adverse events (AEs) were: pyrexia $(63 \%)$ and fatigue $(54 \%)$ (Cohort A); diarrhea (30\%) and rash $(25 \%)$ (Cohort B); and vomiting (67\%) (Cohort C). The overall response rate was $69 \%$ for the 26 BRAF-mutated patients, and apparently does not appear to be higher than the overall response rate of BRAF and MEK inhibitor combinations alone; further follow-up is needed to determine how durable the tumor responses are. Another ongoing phase I/II trial KEYNOTE-022 (https://clinicaltrials.gov/ct2/show/ NCT02130466) is looking at the combination of pembrolizumab $2 \mathrm{mg} / \mathrm{kg}$ Q3W with dabrafenib $150 \mathrm{mg}$ twice daily (BID) and trametinib $2 \mathrm{mg}$ daily (QD). ${ }^{34}$ As of the January 2016 data cutoff, 15 patients were enrolled across the dose determination and dose confirmation arms. DLTs were reported in 3 patients: 1 G4 neutropenia; 1 G4 ALT increased (discontinued); 1 ALT, AST, and gamma-glutamyltransferase increased. All events resolved. Overall, 10 (67\%) patients experienced grade 3-4 treatment-related AEs, with 5 (33\%) discontinuations; there were no treatment-related deaths. Unconfirmed overall response rate was $60 \%$ in 15 patients ( $n=9$ PR, $n=2$ s.d., $n=3 \mathrm{PD}$ ). Based on these results, the recommended phase 2 regimen is pembrolizumab $2 \mathrm{mg} / \mathrm{kg}$ Q3W+dabrafenib $150 \mathrm{mg}$ BID+trametinib $2 \mathrm{mg}$ QD. A phase II randomized study is ongoing.

Finally Sullivan et al. ${ }^{35}$ reported preliminary results of a phase 1 study investigating vemurafenib in combination with atezolizumab, a PD-L1 antibody. Patients with BRAFV600 mutated advanced melanoma received atezolizumab +vemurafenib combination concurrently (cohort $1, n=3$ ) or after a run-in period with vemurafenib alone (56 days for cohort 2 $[n=8]$; 28 days for cohort $3[n=6]$ ). Administration of atezolizumab was intravenous $\mathrm{q} 3 \mathrm{w}$ at $20 \mathrm{mg} / \mathrm{kg}$ (cohort 1) and $15 \mathrm{mg} / \mathrm{kg}$ or $1200 \mathrm{mg}$ fixed (cohort 2 and cohort 3). vemurafenib was administered orally bid $960 \mathrm{mg}$ during runin and $720 \mathrm{mg}$ during the atezolizumab +vemurafenib combination period. Among 19 enrolled patients, 17 were safety and efficacy evaluable. atezolizumab +vemurafenib did not result in any DLTs or unexpected AEs. Incidence of atezolizumab + vemurafenib -related G3 AEs was higher in cohort 1 than in cohort 2 or cohort 3 . AEs were manageable and generally reversible. Overall response rate was higher in cohort $2(75 \%)$ and cohort $3(100 \%)$ vs cohort 1 (33\%). Triple-combination therapy with atezolizumab +vemurafenib and cobimetinib (MEK inhibitor) in this patient population is under investigation (https://clinicaltrials.gov/ct2/show/ NCT01656642). Table 2 summarizes ongoing clinical trials exploring targeted therapies in combination with immunotherapy.

\section{Clinical and Biological Consideration to Combine Targeted Therapy and Immunotherapy}

Traditionally, molecular targeted therapies offer high and rapid responses (days), whereas immune checkpoint inhibitors have slower onset of action (weeks). Combination of BRAF and MEKi represents the treatment of choice in patients with symptomatic disease, for those who deserve rapid response. Similarly, patients with bulky disease would benefit most from combination BRAF and MEKi. Unfortunately, those with elevated baseline lactate dehydrogenase or with brain metastases have only modest additional benefit from the combination of BRAFi and MEKi. Further studies are therefore warranted to identify genomic and immunological factors that contribute to such poor responders.

In patients who do not require rapid symptom control, immune checkpoint inhibitors are another option, given the possibility of durable survival. To address formally the best sequencing, the Intergroup/SWOG Phase III study (https:// clinicaltrials.gov/ct2/show/NCT02224781) is investigating the upfront strategy of dabrafenib-trametinib followed by ipilimumab-nivolumab and vice versa, with both initial treatments given until progression. However, preliminary data raise concern regarding such a clinical design.

The immune response to BRAFi or BRAFi and MEKi happens within 2 weeks but is transient, with very few T cells remaining 4 weeks after initiation of therapy. ${ }^{9,10}$ Hence, in order to optimize recruitment and activation of $\mathrm{T}$ lymphocytes, the induction phase (eg, 2-4 weeks) with BRAFi or BRAFi and MEKi should be followed by the addition of immunotherapy early during the treatment rather than at the time of progression. Furthermore, since the upregulation of PD-L1 in the tumor microenvironment occurs within 24 weeks of initiation of BRAFi, a PD-1-based strategy should be pursued in order to conteract the adaptive resistance. To explore this hypothesis, a three-arm sequencing trial will investigate up front encorafenib-binimetinib followed by ipilimumab-nivolumab at progression or vice versa; while the third arm will explore the activity of encorafenib-binimetinib for 8 weeks followed by ipilimumab-nivolumab before progression (https://clinicaltrials.gov/show/NCT02631447).

Although sequential BRAF-MEKi and immune checkpoint inhibitors are currently in clinical practice, the possibility of concurrent PD-1 and targeted therapy combinations are currently being explored. For instance, the KEYNOTE-022 (https://clinicaltrials.gov/ct2/show/NCT02130466) is looking at the combination of the anti PD-1 pembrolizumab $2 \mathrm{mg} / \mathrm{kg}$ Q3W with dabrafenib $150 \mathrm{mg}$ twice daily (BID) and trametinib $2 \mathrm{mg}$ daily (QD) in order to reverse the immunotolerant microenvironment that emerges early after starting BRAFi or BRAFi and MEKi. ${ }^{34}$ 


\section{CONCLUSIONS AND FUTURE DIRECTIONS}

The systemic treatment of melanoma has evolved with the introduction of BRAFi and MEKi as well as the immune checkpoint inhibitors. Elucidating mechanisms of response and resistance to each of these forms of therapy is critical to better understand how to combine these therapeutic agents. Central to this understanding is translational research conducted in parallel and in sequence in in vitro and murine models as well as in patient samples. It is now clear that in addition to their established molecular mechanism of action, the therapeutic efficacy of BRAFi and MEKi relies on other factors that affect the tumor-host interactions, including the enhancement of melanoma antigen expression and the increase in immune response against tumor cells. Several unanswered questions still remain.

\section{Which Form of Immunotherapy Should be Used in Combination with Targeted Therapy}

Initial and ongoing efforts focused on combining BRAFtargeted therapy with approved agents (such as anti PD-1, PD-L1 antibodies and ipilimumab), but subsequent trials should combine new immune checkpoints such as LAG-3, TIM-3, and IDO, which offer great promise in melanoma and in other cancer types. This introduces two main difficulties: acute and late toxicity as well as long term benefit with these new agents is less known and combination will be challenged by the lack of definitive estimates, that are important for the design of clinical trials.

\section{Which Schedule of Targeted Therapy Should be Used in Combination with Immunotherapy}

It has been argued that continuous dosing of BRAF inhibitors might not be the most efficacious strategy and that intermittent dosing may both delay the development of acquired resistance and delay clinical disease progression once resistant clones have emerged. ${ }^{36,37}$ Exploring the role of intermittent schedule of BRAFi and MEKi with immunotherapy introduced during target therapy holidays will allow shedding the light on the best integration between targeted therapy and immunotherapy.

\section{How to Combine and What Schedule in Cold Melanomas} An important area of investigation is to explore new strategies in melanoma without TILs and PD-L1 expression (immune ignorance). This group represents a not negligible fraction of melanoma patients $(\sim 4 \%)$, with poor prognosis based on their lack of detectable immune reaction. In this group of patients, single-agent checkpoint blockade would most likely not to be successful given the lack of preexisting T-cell infiltrates. In this adverse clinical scenario, BRAFi and MEKi could increase MDAs expression and elicit T-cell activation, bringing $\mathrm{T}$ cells into tumors and then avoiding their being turned off.
DISCLOSURE/CONFLICT OF INTEREST

The authors declare no conflict of interest.

1. Hauschild A, Grob JJ, Demidov LV, et al. Dabrafenib in BRAF-mutated metastatic melanoma: a multicentre, open-label, phase 3 randomised controlled trial. Lancet 2012;380:358-365.

2. Chapman PB, Hauschild A, Robert $C$, et al. Improved survival with vemurafenib in melanoma with BRAF V600E mutation. $N$ Engl J Med 2011;364:2507-2516.

3. Sosman JA, Kim KB, Schuchter $L$, et al. Survival in BRAF V600-mutant advanced melanoma treated with vemurafenib. $\mathrm{N}$ Engl J Med 2012;366:707-714.

4. Flaherty KT, Robert C, Hersey $P$, et al. Improved survival with MEK inhibition in BRAF-mutated melanoma. N Engl J Med 2012;367: 107-114.

5. Robert C, Karaszewska B, Schachter J, et al. Improved overall survival in melanoma with combined dabrafenib and trametinib. N Engl J Med 2015;372:30-39.

6. Long GV, Stroyakovskiy D, Gogas H, et al. Dabrafenib and trametinib versus dabrafenib and placebo for Val600 BRAF-mutant melanoma: a multicentre, double-blind, phase 3 randomised controlled trial. Lancet 2015;386:444-451.

7. Larkin J, Ascierto PA, Dreno B, et al. Combined vemurafenib and cobimetinib in BRAF-mutated melanoma. N Engl J Med 2014;371: 1867-1876.

8. Schadendorf D, Hodi FS, Robert C, et al. Pooled analysis of long-term survival data from phase II and phase III trials of ipilimumab in unresectable or metastatic melanoma. J Clin Oncol 2015;33: 1889-1894.

9. Frederick DT, Piris A, Cogdill AP, et al. BRAF inhibition is associated with enhanced melanoma antigen expression and a more favorable tumor microenvironment in patients with metastatic melanoma. Clin Cancer Res 2013;19:1225-1231.

10. Wilmott JS, Long GV, Howle JR, et al. Selective BRAF inhibitors induce marked T-cell infiltration into human metastatic melanoma. Clin Cancer Res 2012;18:1386-1394.

11. Sumimoto $H$, Imabayashi $F$, Iwata $T$, et al. The BRAF-MAPK signaling pathway is essential for cancer-immune evasion in human melanoma cells. J Exp Med 2006;203:1651-1656.

12. Khalili JS, Liu S, Rodriguez-Cruz TG, et al. Oncogenic BRAF(V600E) promotes stromal cell-mediated immunosuppression via induction of interleukin-1 in melanoma. Clin Cancer Res 2012;18:5329-5340.

13. Berger MF, Hodis E, Heffernan TP, et al. Melanoma genome sequencing reveals frequent PREX2 mutations. Nature 2012;485:502-506.

14. Pleasance ED, Cheetham RK, Stephens PJ, et al. A comprehensive catalogue of somatic mutations from a human cancer genome. Nature 2010;463:191-196.

15. Mandala M, Voit C. Targeting BRAF in melanoma: biological and clinical challenges. Crit Rev Oncol Hematol 2013:87:239-255.

16. Davies $H$, Bignell GR, Cox $C$, et al. Mutations of the BRAF gene in human cancer. Nature 2002;417:949-954.

17. Menzies AM, Haydu LE, Visintin $L$, et al. Distinguishing clinicopathologic features of patients with V600E and V600K BRAF-mutant metastatic melanoma. Clin Cancer Res 2012;18: 3242-3249.

18. Knight DA, Ngiow SF, Li M, et al. Host immunity contributes to the antimelanoma activity of BRAF inhibitors. J Clin Invest 2013;123: 1371-1381.

19. Wargo JA, Cooper ZA, Flaherty KT. Universes collide: combining immunotherapy with targeted therapy for cancer. Cancer Discov 2014;4:1377-1386.

20. Boni A, Cogdill AP, Dang P, et al. Selective BRAFV600E inhibition enhances T-cell recognition of melanoma without affecting lymphocyte function. Cancer Res 2010;70:5213-5219.

21. Comin-Anduix B, Chodon T, Sazegar $H$, et al. The oncogenic BRAF kinase inhibitor PLX4032/RG7204 does not affect the viability or function of human lymphocytes across a wide range of concentrations. Clin Cancer Res 2010;16:6040-6048.

22. Bradley SD, Chen Z, Melendez B, et al. BRAFV600E co-opts a conserved MHC class I internalization pathway to diminish antigen presentation and CD8+ T-cell recognition of melanoma. Cancer Immunol Res 2015;3:602-609. 
23. Sapkota B, Hill CE, Pollack BP. Vemurafenib enhances $\mathrm{MHC}$ induction in BRAFV600E homozygous melanoma cells. Oncoimmunology 2013;2: e22890.

24. Wellbrock C, Rana S, Paterson $\mathrm{H}$, et al. Oncogenic BRAF regulates melanoma proliferation through the lineage specific factor MITF. PLoS One 2008;3:e2734.

25. Ferrari de Andrade L, Ngiow SF, Stannard K, et al. Natural killer cells are essential for the ability of BRAF inhibitors to control BRAFV600Emutant metastatic melanoma. Cancer Res 2014;74:7298-7308.

26. Ebert PJ, Cheung J, Yang Y, et al. MAP kinase inhibition promotes T cell and anti-tumor activity in combination with PD-L1 checkpoint blockade. Immunity 2016;44:609-621.

27. Kakavand H, Wilmott JS, Menzies AM, et al. PD-L1 expression and tumor-infiltrating lymphocytes define different subsets of MAPK inhibitor-treated melanoma patients. Clin Cancer Res 2015;21: 3140-3148.

28. Cooper ZA, Reuben A, Austin-Breneman J, et al. Does it MEK a difference? Understanding immune effects of targeted therapy. Clin Cancer Res 2015;21:3102-3104.

29. Hu-Lieskovan S, Mok S, Homet Moreno B, et al. Improved antitumor activity of immunotherapy with BRAF and MEK inhibitors in BRAF (V600E) melanoma. Sci Transl Med 2015;7:279ra41.

30. Massi D, Brusa D, Merelli B, et al. The status of PD-L1 and tumorinfiltrating immune cells predict resistance and poor prognosis in
BRAFi-treated melanoma patients harboring mutant BRAFV600. Ann Oncol 2015;26:1980-1987.

31. Ribas A, Hodi FS, Callahan M, et al. Hepatotoxicity with combination of vemurafenib and ipilimumab. N Engl J Med 2013;368:1365-1366.

32. Puzanov I, Callahan M, Gerald P, Linette $G$, et al. Phase 1 study of the BRAF inhibitor dabrafenib (D) with or without the MEK inhibitor trametinib ( $T$ ) in combination with ipilimumab (Ipi) for V600E/K mutation-positive unresectable or metastatic melanoma (MM). J Clin Oncol 2014;32.

33. Ribas A, Butler M, Lutzky J, et al. Phase 1 study combining anti-PD-L1 (MEDI4736) with BRAF (dabrafenib) and/or MEK (trametinib) inhibitors in advanced melanoma. J Clin Oncol 2015;33.

34. Ribas A, Hodi F, Lawrence D, et al. Pembrolizumab (pembro) in combination with dabrafenib (D) and trametinib (T) for BRAF-mutant advanced melanoma: phase 1 KEYNOTE-022 study. J Clin Oncol 2016;34.

35. Sullivan R, Hamid $O$, Patel $M$, et al. Melanoma and immunotherapy bridge 2015. Naples, Italy. 1-5 December 2015. J Transl Med 2016;14:65.

36. Shi $\mathrm{H}$, Hugo $W$, Kong $X$, et al. Acquired resistance and clonal evolution in melanoma during BRAF inhibitor therapy. Cancer Discov 2014;4: 80-93.

37. Das Thakur M, Salangsang F, Landman AS, et al. Modelling vemurafenib resistance in melanoma reveals a strategy to forestal drug resistance. Nature 2013;494:251-255. 